\title{
SIMILARITY OF CERTAIN OPERATORS IN $l^{p}$
}

\author{
SHMUEL KANTOROVITZ ${ }^{1}$
}

ABstract. Let $M$ be the multiplication operator in $l^{p}, 1<p<\infty$, i.e., $M$ : $x=\left\{x_{k}\right\} \rightarrow\left\{k x_{k}\right\}$. Let $w=\left\{w_{j}\right\}_{j=0}^{\infty}$ be a weight, i.e., a positive sequence such that $w_{1}<w_{0}=1$ and $w_{n+m} \leqslant w_{n} w_{m}$. For $\zeta \in C$, define $N_{w}^{\zeta}$ on $l^{p}$ by

$$
\left(N_{w}^{\zeta} x\right)_{k}=\sum_{j=1}^{k}\left(\frac{w_{k}}{w_{j}}\right)\left(\begin{array}{c}
\zeta-1+k-j \\
k-j
\end{array}\right) x_{j} \quad(k=1,2, \ldots) .
$$

Then $\left\{N_{w}^{\zeta} ; \zeta \in C\right\}$ is a holomorphic group of operators, and for any function $g$ holomorphic on the spectrum of $N_{w}^{\zeta}, M+g\left(N_{w}^{\zeta}\right)$ is similar to $M+g(1) I$.

1. Introduction. It was proved in [3] that the operators $M+\lambda J$ and $M+\mu J$ in $L^{p}(0,1), 1<p<\infty$ (where $M: f(x) \rightarrow x f(x)$ and $J: f(x) \rightarrow \int_{0}^{x} f(t) d t ; \lambda$, $\mu \in C)$ are similar if and only if $\operatorname{Re} \lambda=\operatorname{Re} \mu$. It is natural to look for an analogous result in $l^{p}$, with $M$ the "multiplication operator"

$$
(M x)_{k}=k x_{k} \quad\left(x=\left\{x_{k}\right\}_{k=1}^{\infty} \in l^{p}\right),
$$

and with a suitable discrete analog of $J$. Since $M$ is an unbounded (closed, densely defined) operator in $l^{p}$, it is convenient to have the corresponding $J$ bounded, and the natural choice is the "weighted summation operator" in $l^{p}$

$$
\left(N_{w} x\right)_{k}=\sum_{j=1}^{k}\left(w_{k} / w_{j}\right) x_{j} \quad(k=1,2, \ldots),
$$

where the "weight" $w=\left\{w_{j}\right\}_{j=0}^{\infty}$ is a positive sequence such that

$$
\begin{gathered}
w_{1}<w_{0}=1, \\
w_{n+m} \leqslant w_{n} w_{m} \quad(m, n=0,1,2, \ldots) .
\end{gathered}
$$

For example, $w_{n}=\exp \left(-a n^{b}\right)$ defines a weight for $a>0, b \geqslant 1$. On the other hand, any weight $w$ is majorized by an exponential weight $\left\{e^{-a n}\right\}$, since $w_{n} \leqslant w_{1}^{n}$ by (3) and $w_{1}=e^{-a}$ with $a>0$ by (2). Note also that $w$ is strictly decreasing $\left(w_{n+m} \leqslant w_{n} w_{1}^{m}<w_{n}\right)$, and $\|w\|=\sum_{n=0}^{\infty} w_{n} \leqslant\left(1-w_{1}\right)^{-1}$.

The result in [3], and a corresponding result for $L^{p}(0, \infty)$ [7, p. 1196] (which is a closer analog to our $l^{p}$ situation) have been imbedded in an abstract theory [4], [7] whose main ingredients are:

(i) $J$ is imbedded in a "regular" semigroup of operators;

Received by the editors December 15, 1976.

AMS (MOS) subject classifications (1970). Primary 47A65, 47A60; Secondary 47B40.

Key words and phrases. Holomorphic group of operators, closed operator, similarity, spectral operator.

${ }^{1}$ Research partially supported by NSF Grant No. MCS76-07496.

(1) American Mathematical Society 1977 
(ii) the pair $(M, J)$ satisfies the "Volterra commutation relation" $[M$, $J] \subset J^{2}$ (where $\left.[M, J]=M J-J M\right)$.

Looking first for an enveloping semigroup for our $N_{w}$, we actually discover an enveloping holomorphic group $\left\{N_{w}^{\zeta} ; \zeta \in C\right\}$ given by

$$
\left(N_{w}^{\zeta} x\right)_{k}=\sum_{j=1}^{k}\left(\frac{w_{k}}{w_{j}}\right)\left(\begin{array}{c}
\zeta-1+k-j \\
k-j
\end{array}\right) x_{j} \quad\left(x \in l^{p}\right),
$$

where

$$
\left(\begin{array}{l}
\alpha \\
n
\end{array}\right)=\alpha(\alpha-1) \cdots(\alpha-n+1) / n ! \text { for } n=1,2, \ldots
$$

and

$$
\left(\begin{array}{l}
\alpha \\
0
\end{array}\right)=1 \quad(\alpha \in C) .
$$

Trivially, $N_{w}^{1}=N_{w}, N_{w}^{0}=I$ (the identity operator), and $\left(N_{w}^{\zeta} x\right)_{1}=x_{1}$ for all $\zeta \in C$. However, instead of (ii), we have $\left[M, N_{w}\right] \subset N_{w}\left(N_{w}-I\right)$, and this "slight" difference leads to totally different results: all the operators $M+$ $\lambda N_{w}^{\zeta}$ are similar to $M+\lambda I$; in particular, $M+\lambda N_{w_{1}}^{\zeta}$ is similar to $M+\mu N_{w_{2}}^{\omega}$ if and only if $\lambda=\mu$ !. More generally, our main result (Theorem 2) states that for any weight $w$, for any $\zeta \in C$, and for any function $g$ holomorphic on the spectrum $\sigma\left(N_{w}^{\zeta}\right)$ of $N_{w}^{\zeta}$, the operator $M+g\left(N_{w}^{\zeta}\right)$ is similar to $M+g(1) I$. Since $M+\lambda I$ and $M+\mu I$ are similar if and only if $\lambda=\mu$ (by spectrum consideration), our theorem establishes that within the family $\mathfrak{N}=\{\boldsymbol{M}+$ $\left.g\left(N_{w}^{\zeta}\right)\right\}$ (where the weight $w$, the complex number $\zeta$, and the function $g$ holomorphic on $\sigma\left(N_{w}^{\zeta}\right)$ are parameters), a complete set of similarity invariants is $\{g(1)\}$, and $M+g(1) I$ is a canonical similarity model for the class with the invariant $g(1)$. Note that $\mathfrak{T}$ is a family of spectral operators of scalar type (since $M+\lambda I$ is trivially of this type). This should be compared to the negative results for $\operatorname{Re} \zeta=1$ in $L^{p}$ (cf. [3], [5, Theorem 12], [7]), and to the positive results for $\operatorname{Re} \zeta>1$ there (cf. $[4, \S 7]$ and [7]).

In this paper, we say that a function is holomorphic "on $K$ " (with $K$ compact) if it is holomorphic in a simply connected open set containing $K$.

\section{The enveloping group.}

THEOREM 1. For each weight $w$ and $1 \leqslant p \leqslant \infty,\left\{N_{w}^{\zeta} ; \zeta \in C\right\}$ is a holomorphic group of bounded operators in $l^{p}$. Its infinitesimal generator is the bounded operator $A_{w}$ defined by

$$
\left(A_{w} x\right)_{1}=0, \quad\left(A_{w} x\right)_{k}=\sum_{j=1}^{k-1}\left(\frac{w_{k}}{w_{j}}\right) \frac{x_{j}}{k-j} \quad(k>1) .
$$

In particular,

$$
N_{w}^{\zeta}=\exp \left(\zeta A_{w}\right), \quad \zeta \in C .
$$

Proof. Since $w_{k} / w_{j} \leqslant w_{k-j}(0 \leqslant j \leqslant k)$ by (3), and

$$
\left|\left(\begin{array}{c}
\zeta-1+j \\
j
\end{array}\right)\right| \leqslant\left(\begin{array}{c}
|\zeta|-1+j \\
j
\end{array}\right) \quad(\zeta \in C ; j=0,1,2, \ldots) \text {, }
$$


we have

$$
\left|\left(N_{w}^{\zeta} x\right)_{k}\right| \leqslant \sum_{j=1}^{k} w_{k-j}\left(\begin{array}{c}
|\zeta|-1+k-j \\
k-j
\end{array}\right)\left|x_{j}\right| \quad\left(x \in l^{p}\right)
$$

Let

$$
\left(k_{w}^{\zeta}\right)_{j}=\left\{\begin{array}{l}
0, \quad j<0, \\
w_{j}\left(\begin{array}{c}
\zeta-1+j \\
j
\end{array}\right), \quad j \geqslant 0,
\end{array}\right.
$$

and $(|x|)_{k}=\left|x_{k}\right|$. Considering $l^{p}$ as a subspace of $l^{p}(Z)$, the right-hand side of (7) is the convolution $k_{w}^{|\zeta|} *|x|$, and it follows that

$$
\left\|N_{w}^{\zeta} x\right\|_{p} \leqslant\left\|k_{w}^{|\zeta|}\right\|_{1}\|x\|_{p}
$$

Since

$$
\left\|k_{w}^{|\xi|}\right\|_{1} \leqslant \sum_{j=0}^{\infty}\left(\begin{array}{c}
|\zeta|-1+j \\
j
\end{array}\right) w_{1}^{j}=\left(1-w_{1}\right)^{-|\zeta|}
$$

we conclude that for each weight $w$ and $\zeta \in C$,

$$
\left\|N_{w}^{\zeta}\right\| \leqslant\left(1-w_{1}\right)^{-|\xi|}
$$

The group property is verified next. Fix $x \in l^{p}$, and $\alpha, \beta \in C$. For $n=1$, $2, \ldots$,

$$
\begin{aligned}
\left(N_{w}^{\alpha} N_{w}^{\beta} x\right)_{n} & =\sum_{k=1}^{n}\left(\frac{w_{n}}{w_{k}}\right)\left(\begin{array}{c}
\alpha-1+n-k \\
n-k
\end{array}\right) \sum_{j=1}^{k}\left(\frac{w_{k}}{w_{j}}\right)\left(\begin{array}{c}
\beta-1+k-j \\
k-j
\end{array}\right) x_{j} \\
& =\sum_{j=1}^{n}\left(\frac{w_{n}}{w_{j}}\right) \sum_{r=0}^{n-j}\left(\begin{array}{c}
\alpha-1+n-j-r \\
n-j-r
\end{array}\right)\left(\begin{array}{c}
\beta-1+r \\
r
\end{array}\right) x_{j} \\
& =\sum_{j=1}^{n}\left(\frac{w_{n}}{w_{j}}\right)\left(\begin{array}{c}
\alpha+\beta-1+n-j \\
n-j
\end{array}\right) x_{j} \\
& =\left(N_{w}^{\alpha+\beta} x\right)_{n} .
\end{aligned}
$$

In the transition from the second to the third line, we used the identity

$$
\sum_{r=0}^{m}\left(\begin{array}{c}
\alpha-1+m-r \\
m-r
\end{array}\right)\left(\begin{array}{c}
\beta-1+r \\
r
\end{array}\right)=\left(\begin{array}{c}
\alpha+\beta-1+m \\
m
\end{array}\right)
$$

which follows trivially from the binomial expansion

$$
(1-x)^{-\alpha}=\sum_{j=0}^{\infty}\left(\begin{array}{c}
\alpha-1+j \\
j
\end{array}\right) x^{j} \quad(|x|<1)
$$

and the convolution formula for the coefficients of the product of power series.

Consider now the operator $A_{w}$ defined in (5). Regarding again $l^{p}$ as a subspace of $l^{p}(Z)$, we have 


$$
\left|\left(A_{w} x\right)_{k}\right| \leqslant\left(a_{w} *|x|\right)_{k}
$$

for all $x \in l^{p}$, where $\left(a_{w}\right)_{j}=0$ for $j \leqslant 0$ and $\left(a_{w}\right)_{j}=w_{j} / j$ for $j \geqslant 1$. Hence

$$
\left\|A_{w}\right\| \leqslant\left\|a_{w}\right\|_{1} \leqslant \sum_{j=1}^{\infty} \frac{w_{1}^{j}}{j}=-\log \left(1-w_{1}\right),
$$

proving the boundedness of $A_{w}$.

For $\zeta \in C \backslash\{0\}$, let $T_{w}^{\zeta}=\zeta^{-1}\left(N_{w}^{\zeta}-I\right)-A_{w}$. Then for each $x \in l^{p}$, $\left(T_{w}^{\zeta} x\right)_{k}=0$ for $k=1,2$, and

$$
\left(T_{w}^{\zeta} x\right)_{k}=\sum_{j=1}^{k-2}\left(\frac{w_{k}}{w_{j}}\right) \frac{[(\zeta+1) \cdots(\zeta+k-j-1)-(k-j-1) !] x_{j}}{(k-j) !}
$$

for $k>2$. It follows that

$$
\left|\left(T_{w}^{\zeta} x\right)_{k}\right| \leqslant\left(h_{w}^{\zeta} *|x|\right)_{k} \quad(k=1,2, \ldots)
$$

where $\left(h_{w}^{\zeta}\right)_{k}=0$ for $k \leqslant 1$ and

$$
\left(h_{w}^{\zeta}\right)_{k}=w_{k}|(\zeta+1) \cdots(\zeta+k-1)-(k-1) !| / k ! \text { for } k>1
$$

(as usual, $l^{p}$ is considered as a subspace of $l^{p}(Z)$ ). Hence $\left\|T_{w}^{\zeta}\right\| \leqslant\left\|h_{w}^{\zeta}\right\|_{1}$. For $|\zeta| \leqslant 1$, the series for $\left\|h_{w}^{\zeta}\right\|_{1}$ is majorized by the convergent series $2 \sum_{k=2}^{\infty} w_{1}^{k}$. Therefore $\left\|h_{w}^{\zeta}\right\|_{1}$ is a continuous function of $\zeta$ in $|\zeta| \leqslant 1$, and since $\left\|h_{w}^{0}\right\|_{1}=0$, it follows that $\left\|h_{w}^{\zeta}\right\|_{1} \rightarrow 0$ as $\zeta \rightarrow 0$, and we conclude that $\lim _{\zeta \rightarrow 0} \zeta^{-1}\left(N_{w}^{\zeta}-I\right.$. $=A_{w}$ in the uniform operator topology. Since $\left\{N_{w}^{\zeta} ; \zeta \in C\right\}$ is a group, the proof is complete.

3. The similarity result. We come now to our main result, valid in any $l^{p}$ $(1 \leqslant p \leqslant \infty)$ and for any weight $w$.

THEOREM 2. For any $\zeta \in C$ and for any complex function $g$ holomorphic on $\sigma\left(N_{w}^{\zeta}\right), M+g\left(N_{w}^{\zeta}\right)$ is similar to $M+g(1) I$.

PROof. We first reduce the general case to the case $\zeta=1$.

As in the proof of Theorem 1, one obtains the estimate

$$
\left\|N_{w}^{i t}\right\| \leqslant \sum_{j=0}^{\infty} w_{j}\left|\left(\begin{array}{c}
i t-1+j \\
j
\end{array}\right)\right|
$$

For $j \geqslant 1$,

$$
\left(\begin{array}{c}
i t-1+j \\
j
\end{array}\right)=\prod_{m=1}^{j-1}\left(1+\frac{i t}{m}\right) \cdot \frac{i t}{j}
$$

(where $\Pi_{1}^{0}=1$ ); hence

$$
\begin{aligned}
\left|\left(\begin{array}{c}
i t-1+j \\
j
\end{array}\right)\right|^{2} & =\prod_{m=1}^{j-1}\left(1+\frac{t^{2}}{m^{2}}\right) \cdot\left(\frac{t^{2}}{j^{2}}\right) \\
& <\prod_{m=1}^{\infty}\left(1+\frac{t^{2}}{m^{2}}\right)=\frac{\sinh \pi t}{\pi t}
\end{aligned}
$$


Therefore,

$$
\left\|N_{w}^{i t}\right\|<\|w\|(\sinh \pi t / \pi t)^{1 / 2}
$$

Let

$$
s^{+}\left(A_{w}\right)=\sup \operatorname{Im} \sigma\left(A_{w}\right) \text { and } s^{-},\left(A_{w}\right)=\inf \operatorname{Im} \sigma\left(A_{w}\right) \text {. }
$$

By $[2$, p. 165],

$$
\begin{aligned}
s^{ \pm}\left(A_{w}\right) & =\lim _{t \rightarrow \pm \infty} t^{-1} \log \left\|\exp \left(-i t A_{w}\right)\right\| \\
& =\lim _{t \rightarrow \pm \infty} t^{-1} \log \left\|N_{w}^{-i t}\right\|,
\end{aligned}
$$

and by (10),

$$
\left|s^{ \pm}\left(A_{w}\right)\right| \leqslant \frac{1}{2} \lim _{|t| \rightarrow \infty}|t|^{-1} \log |\sinh \pi t|=\pi / 2 .
$$

Since $\sigma\left(N_{w}\right)=\exp \sigma\left(A_{w}\right)$, it follows that

$$
\left|\operatorname{Arg} \sigma\left(N_{w}\right)\right|=\left|\operatorname{Im} \sigma\left(A_{w}\right)\right| \leqslant \pi / 2,
$$

i.e., $\sigma\left(N_{w}\right)$ is contained in the closed right half-plane, and since $0 \notin \sigma\left(N_{w}\right)$ (cf. Theorem 1), the nonpositive axis is not in $\sigma\left(N_{w}\right)$. Therefore the principal values $\log \lambda$ and $h_{\zeta}(\lambda)=\lambda^{\zeta}=\exp (\zeta \log \lambda$ ) (for any $\zeta \in C$ ) are holomorphic on $\sigma\left(N_{w}\right)$. By the composite function theorem [1, p. 171],

$$
\log N_{w}=\log \exp \left(A_{w}\right)=A_{w}
$$

and

$$
h_{\zeta}\left(N_{w}\right)=\exp \left(\zeta \log N_{w}\right)=\exp \left(\zeta A_{w}\right)=N_{w}^{\zeta} .
$$

Let $g$ be a complex function holomorphic on $\sigma\left(N_{w}^{\zeta}\right)=h_{\zeta}\left(\sigma\left(N_{w}\right)\right)$. Then $f_{\zeta}=g \circ h_{\zeta}$ is holomorphic on $\sigma\left(N_{w}\right)$ and $f_{\zeta}\left(N_{w}\right)=g\left(N_{w}^{\zeta}\right)$. Assuming Theorem 2 for $\zeta=1$, we obtain that $M+g\left(N_{w}^{\zeta}\right)=M+f_{\zeta}\left(N_{w}\right)$ is similar to $M+$ $f_{\zeta}(1) I=M+g(1) I$, and the general case is established.

It remains to prove Theorem 2 for $\zeta=1$. We rely on the following special case of our theorem in [6].

LEMMA. Let $B$ and $C$ be commuting bounded operators in a Banach space $X$, and let $M$ be a closed operator in $X$ with $B$-invariant domain such that $[M$, $B] \subset C$. Then for any complex function $f$ holomorphic on $\sigma(B), M+f^{\prime}(B) C$ is similar to M. More precisely,

$$
M+f^{\prime}(B) C=e^{-f(B)} M e^{f(B)}
$$

(as unbounded operators).

In our case, a direct calculation shows that the multiplication operator $M$ in $l^{p}$ has $A_{w}$-invariant domain, and $\left[M, A_{w}\right] \subset N_{w}-I$. By the lemma,

$$
M+f^{\prime}\left(A_{w}\right)\left(N_{w}-I\right)=\exp \left[-f\left(A_{w}\right)\right] M \exp f\left(A_{w}\right)
$$

for any complex function $f$ holomorphic on $\sigma\left(A_{w}\right)$. If $h$ is holomorphic on $\sigma\left(N_{w}\right), f(\lambda)=h\left(e^{\lambda}\right)$ is holomorphic on $\sigma\left(A_{w}\right)$, and (12) implies 


$$
M+h^{\prime}\left(N_{w}\right) N_{w}\left(N_{w}-I\right)=\exp \left[-h\left(N_{w}\right)\right] M \exp h\left(N_{w}\right) .
$$

Since $\left(A_{w} x\right)_{1}=0$ for all $x \in l^{p}, A_{w}$ is not onto, hence $0 \in \sigma\left(A_{w}\right)$, and therefore $1 \in \sigma\left(N_{w}\right)$. Consequently, any $g$ holomorphic on $\sigma\left(N_{w}\right)$ may be written as

$$
g(\lambda)=g(1)+(\lambda-1) g_{1}(\lambda)
$$

with $g_{1}$ holomorphic on $\sigma\left(N_{w}\right)$. Since $0 \notin \sigma\left(N_{w}\right), g_{1}(\lambda) / \lambda$ is holomorphic on $\sigma\left(N_{w}\right)$, and there exists a function $h$ holomorphic on $\sigma\left(N_{w}\right)$ such that $h^{\prime}(\lambda)=$ $g_{1}(\lambda) / \lambda$. We have

$$
h^{\prime}(\lambda) \lambda(\lambda-1)=g(\lambda)-g(1),
$$

and therefore (13) implies

$$
M+g\left(N_{w}\right)-g(1) I=\exp \left[-h\left(N_{w}\right)\right] M \exp h\left(N_{w}\right),
$$

i.e.,

$$
M+g\left(N_{w}\right)=\exp \left[-h\left(N_{w}\right)\right][M+g(1) I] \exp h\left(N_{w}\right) .
$$

Q.E.D.

\section{REFERENCES}

1. E. Hille and R. S. Phillips, Functional analysis and semi-groups, rev. ed., Amer. Math. Soc. Colloq. Publ., vol. 31, Amer. Math. Soc., Providence, R. I., 1957.

2. S. Kantorovitz, On the characterization of spectral operators, Trans. Amer. Math. Soc. 111 (1964), 152-181.

3., The $C^{k}$-classification of certain operators in $L_{p}$. II, Trans. Amer. Math. Soc. 146 (1969), 61-68.

4. Commutators, $C^{k}$-classification, and similarity of operators, Trans. Amer. Math. Soc. 156 (1971), 193-218.

5. __ Spectral equivalence and Volterra elements, Indiana Univ. Math. J. 22 (1973), 951-957.

6. Commutation de Heisenberg-Volterra et similarité de certaines perturbations, C. R. Acad. Sci. Paris 276 (1973), 1501-1504.

7. S. Kantorovitz and K. J. Pei, Pairs of operators satisfying the Volterra commutation relation, Indiana Univ. Math. J. 23 (1974), 1177-1197.

Department of Mathematics, University of Illinois at Chicago Circle, Chicago, ILLINOIS 60680

Current address: Department of Mathematics, Bar-Ilan University, Ramat-Gan, Israel 